\title{
The Expected Angular Resolution Performance of the Tilted JEM-EUSO Instrument
}

Thomas Mernik*, Alejandro Guzman, Andrea Santangelo, Kenji Shinozaki, Jörg Bayer, Elias Iwotschkin

Institut für Astronomie und Astrophysik, Eberhard Karls Universität Tübingen, Germany

E-mail: mernik@astro.uni-tuebingen.de

Mario Bertaina, Francesco Fenu

Università degli Studi di Torino, Italy

for the JEM-EUSO Collaboration

JEM-EUSO (The Extreme Universe Observatory onboard the Japanese Experiment Module) is a space borne UV-telescope for the observation of UHECR induced extensive air showers (EAS). Currently in the development phase it will be attached to the ISS (International Space Station) to use the earth's atmosphere as a large detector. Due to the large target volume it gains an effective aperture of approx. $2 \times 10^{5} \mathrm{~km}^{2} \mathrm{sr}$ - sufficient for the CR observation above an energy of $3 \times 10^{19} \mathrm{eV}$. During the mission lifetime, JEM-EUSO will observe several hundred of events above $\mathrm{E}=5 \times 10^{19} \mathrm{eV}$, significantly improving the statistics in this part of the UHECR spectrum. The default operation mode of the instrument is envisaged to be along its nadir direction. However, in a later stage of the mission, a tilting of the telescope, away from its nadir direction, is discussed as a potential strategy to further increase the exposure at the highest energies. In the tilted mode operation the exposure would significantly increase. Hence, the regime of extreme energies above $10^{20} \mathrm{eV}$ could be explored in a reasonable amount of time. Naturally, in this setup the angular resolution of the instrument is expected to decrease. In the scope of this work we evaluate the expected angular resolution performance of the JEM-EUSO instrument in dependence of the tilting angle.

The 34th International Cosmic Ray Conference,

30 July- 6 August, 2015

The Hague, The Netherlands

\footnotetext{
* Speaker.
} 


\section{JEM-EUSO Orbit altitude:}

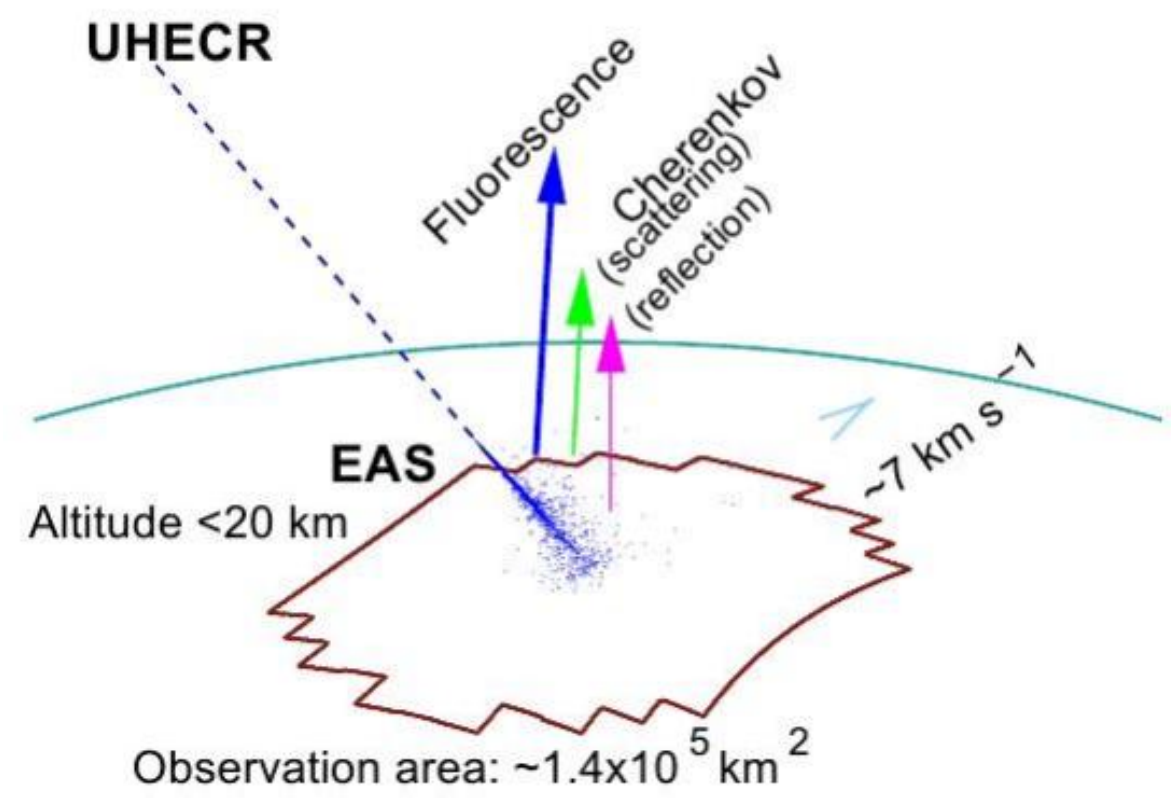

Figure 1: Concept of the JEM-EUSO mission [3].

\section{The JEM-EUSO Mission}

JEM-EUSO, the Extreme Universe Space Observatory onboard the Japanese Experiment Mod$u l e$, is a space-borne UHECR telescope in the design phase. Its primary objective is the observation of UHECR induced air showers from space by taking advantage of the earth's atmosphere. Due to the large target volume monitored, JEM-EUSO will be able to measure cosmic rays with energies around the GZK cut-off $[1,2]$ to a sufficient extent. The detector itself collects the fluorescence and Cherenkov photons that are emitted from the developing showers in the wavelength range between 300 to $400 \mathrm{~nm}$ (Fig. 1). The mission will have a duration of at least $3+2$ years. During this time JEM-EUSO is expected to detect several hundred events exceeding the energy of $5 \times 10^{19} \mathrm{eV}$ [4]. Hence, it will increase the statistics in the high energy part of the spectrum by a factor of ten. At the altitude of the ISS at approximately $400 \mathrm{~km}$, the telescope moves at a speed of about $7 \mathrm{~km} / \mathrm{s}$ in the latitude range of $\pm 51^{\circ}$ [5]. The orbit provides a homogeneous exposure to both hemispheres. The UV telescope comprises an optical unit to focus the incoming photons and a focal surface detector (FSD). The FSD is a collection of photo-detection modules (PDMs) and their corresponding readout electronics. Each PDM is made of a set of multi-anode photomultiplier tubes (MAPMT). What is crucial for this setup is a high time resolution and the single photon count ability. A dedicated, multi-stage trigger algorithm identifies potential CR events from other light sources (atmospheric phenomena or human-induced). The signal characteristics allow for the reconstruction of the properties of the primary UHECR like the particle type, its energy and direction. Being the successor of 
the formerly planned (ESA-) EUSO mission, JEM-EUSO's overall concept has remained similar, but differs in some important aspects. The overall performance of the telescope has significantly evolved. The improved optical design, new lens materials and advanced photomultipliers featuring a higher quantum efficiency decrease the energy threshold to $3 \times 10^{19} \mathrm{eV}$. This allows for cross calibration with nowadays ground based UHECR observatories.

Although, JEM-EUSO mission will primarily observe aligned to the direction of its nadir point, it is envisaged to be tilted away from its nadir axis to increase the already large detection area even further by some factor. The idea is to compensate for potential losses of observation area due to lower space station orbits (this will mostly be achieved by small tilting angles), but also to substantially enlarge the observation area depending on the scientific objective. Depending on the tilting angle, the telescope can reach an instantaneous aperture between 65 and 280 times larger than the Pierre Auger Observatory (Fig. 2). It corresponds to an observational area of $2 \times 10^{5} \mathrm{~km}^{2}$ in nadir and $7 \times 10^{5} \mathrm{~km}^{2}$ in tilted mode [6]. This could yield a sufficient exposure even for the observation of UHE neutrinos. On the downside, due to the tilting fewer photons per event will
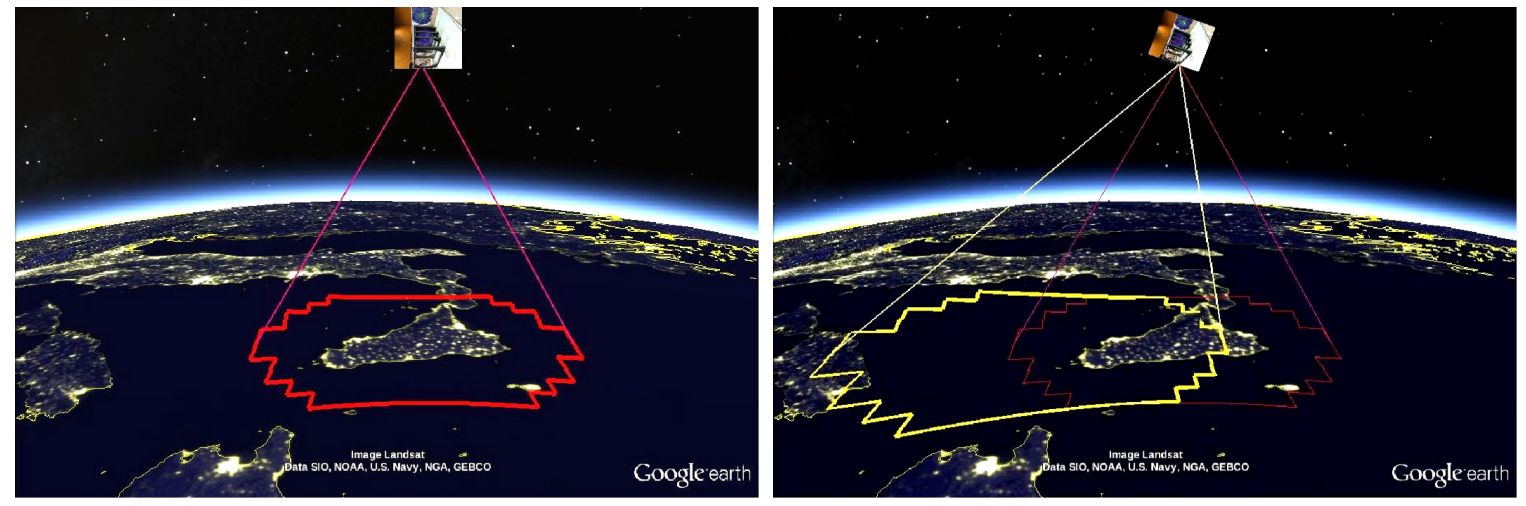

Figure 2: JEM-EUSO field of view over the Mediterranean sea [7]. Left: Viewing direction aligned to the nadir axis, Right: The instrument is tilted away from the nadir direction for an enlarged field of view.

reach the telescope. Therefore, the energy threshold would increase and the reconstruction quality of the measured events is expected to suffer. In this paper we have conducted an extensive study to quantify the effect on the angular resolution for a $20^{\circ}$ and a $40^{\circ}$ tilting of the instrument.

\section{Tilted Mode Studies}

\subsection{Signal Behaviour}

To analyse how the signal changes when we tilt the telescope by $20^{\circ}$ and by $40^{\circ}$ from the nadir direction, we simulated air showers with a fixed energy $\left(10^{20} \mathrm{eV}\right)$ and zenith angle $\left(\Theta=60^{\circ}\right)$ to use it as a standard candle. The simulations are done with the ESAF software ${ }^{1}$. The brightness of these showers is well defined when we use a parametrised shower simulation approach combination with a parametrised photon propagator in the atmosphere. We injected some ten thousand showers

\footnotetext{
${ }^{1}$ The EUSO Simulation and Analysis Framework has been developed by the EUSO collaboration [8]
} 
homogeneously inside an area larger than the field of view of the telescope and studied how many photons per event arrive at the telescope, pass through the optics make a signal on the focal surface detector and create a signal. We can clearly observe that the showers appear brighter when they
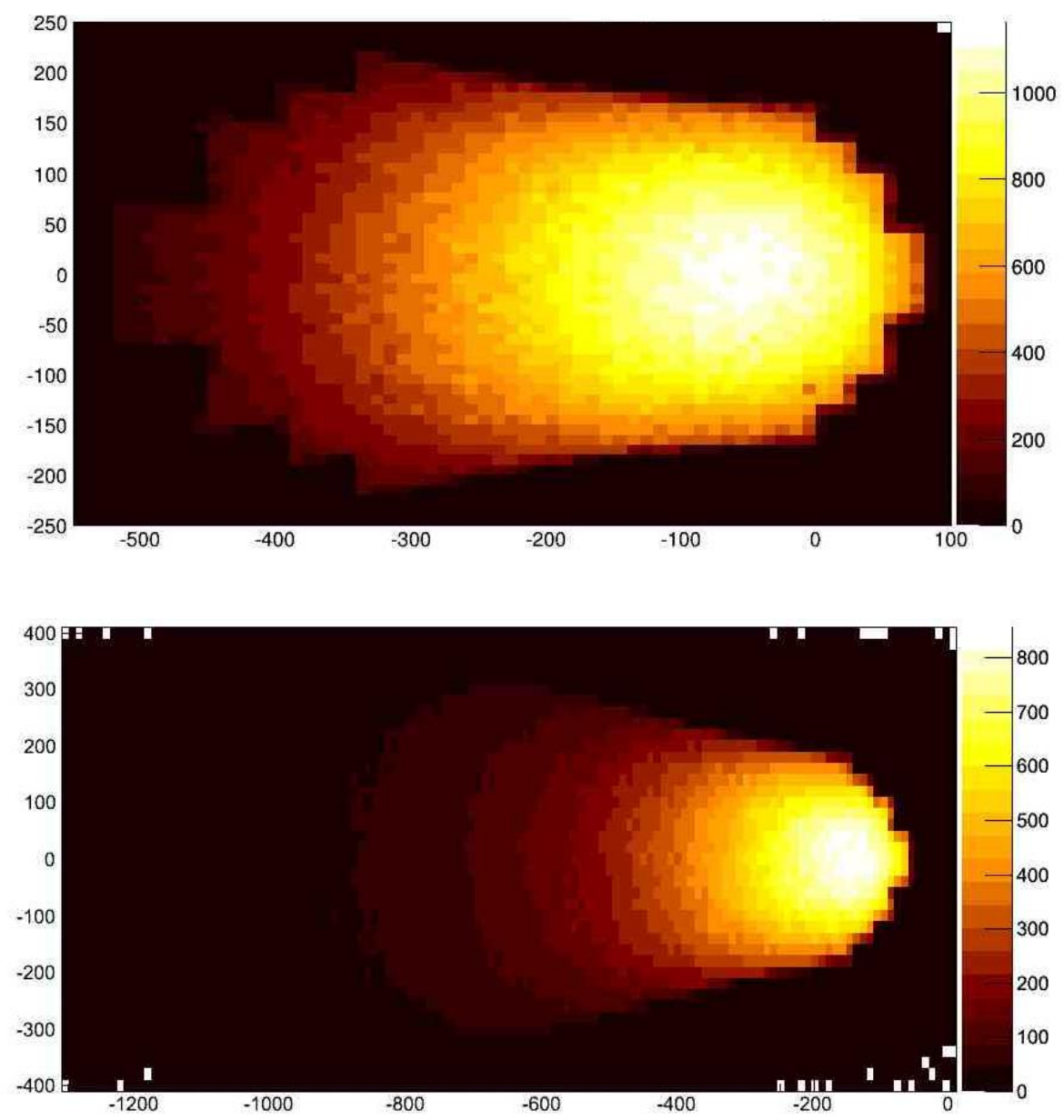

Figure 3: The distribution of photon counts per shower in the FOV (in $\mathrm{km}$ ) of the tilted instrument for $20^{\circ}$ (upper) and for the $40^{\circ}$ case (lower) [7]. Note the different scales.

are simulated closer to the nadir point of the detector (Fig. 3). If injected farther away from the detector, fewer signal per event are created in the focal surface detector. If the number of signals per shower drops below 100 to 200 counts, the shower is very unlikely to be triggered at all. This imposes a certain limit on the effective area of the tilted instrument, which strongly depends on the energy and zenith angle of the shower. A shower with a sufficiently high energy or a strong inclination might produce enough photons to be clearly observed even at the edge of the tilted FOV.

\subsection{Angular Resolution}

To evaluate the expected angular resolution performance of the tilted instrument we produced a database of proton-induced air showers with five different fixed energies and four different fixed zenith angles. The energy values comprise $5 \times 10^{19} \mathrm{eV}, 7 \times 10^{19} \mathrm{eV}, 1 \times 10^{20} \mathrm{eV}, 3 \times 10^{20} \mathrm{eV}$ to $5 \times 10^{20} \mathrm{eV}$, the zenith angles include $30^{\circ}, 45^{\circ}, 60^{\circ}$ and $75^{\circ}$. All azimuth angles are picked randomly 


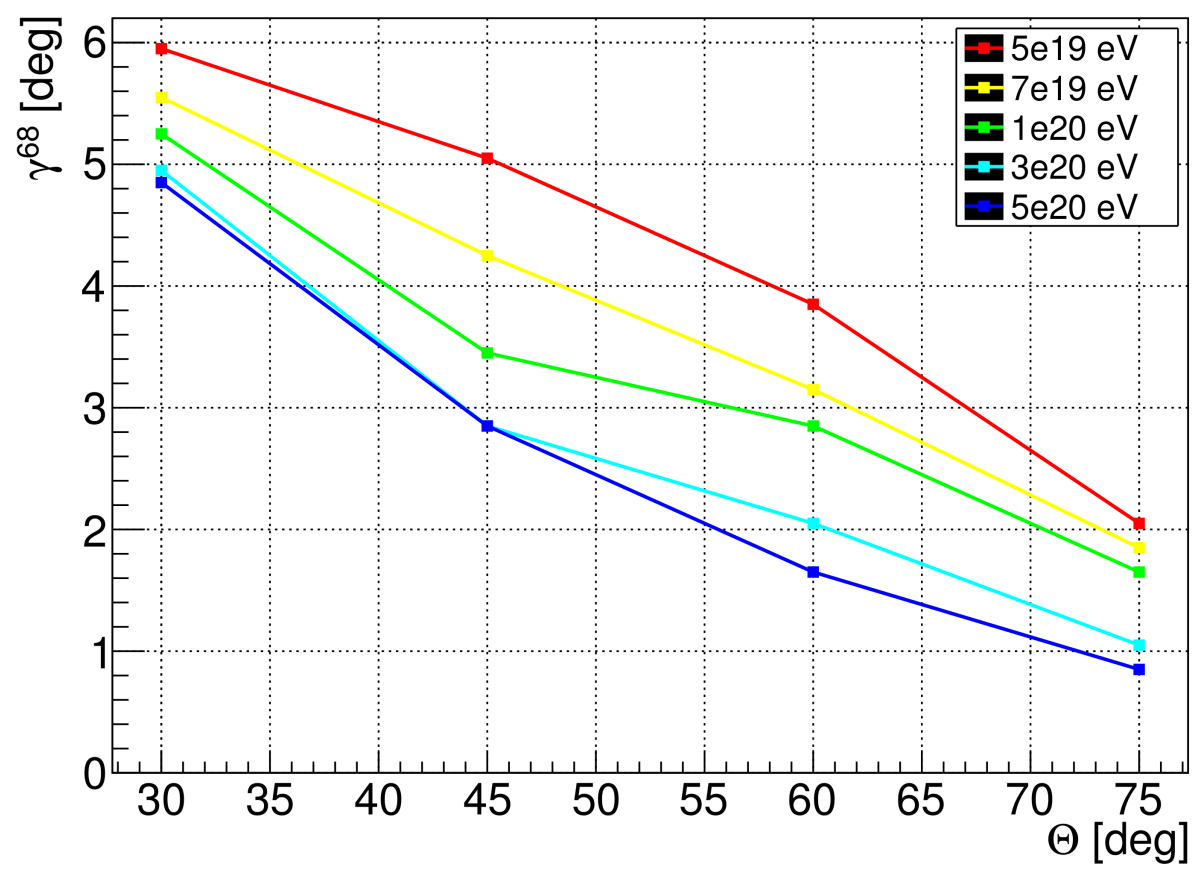

Figure 4: The angular resolution of the tilted $\left(20^{\circ}\right)$ JEM-EUSO telescope. $\gamma$ denotes the angle between the true and the reconstructed direction. $\gamma^{68}$ indicates the cumulative distribution. [7]

between 0 and $360^{\circ}$. The shower cores have been placed within a rectangular area of $\mathrm{x}:-550 \mathrm{~km}-$ $+100 \mathrm{~km} \times \mathrm{y}:-250 \mathrm{~km}-+250 \mathrm{~km}\left(20^{\circ}\right)$ and $\mathrm{x}:-1300 \mathrm{~km}-0 \mathrm{~km} \times \mathrm{y}:-400 \mathrm{~km}-+400 \mathrm{~km}\left(40^{\circ}\right)$ This is well larger than the actual FOV of the tilted instrument. For each of the energy/zenith angle combinations the amount of triggering events is of the order of 2000 or higher. As expected, the angular resolution of the tilted $\left(20^{\circ}\right)$ telescope decreases by approximately $1^{\circ}$ when compared to the nadir mode operation ${ }^{2}$ (Fig. 4). The effect mainly depends on the zenith angle of the showers and to a smaller extent on the energy. The low zenith angle showers loose about $1.5^{\circ}$ in resolution.

When we tilt the telescope by $40^{\circ}$, the resolution decreases compared to the previous $20^{\circ}$ case. Again, the effect mainly depends on the zenith angle of the showers and to a smaller extent on the energy. The loss of the angular resolution is about $1.5^{\circ}$ compared to the tilted $\left(20^{\circ}\right)$ case. (Fig. 5) To check the reason behind the significant worsening of the angular resolution, we examined the dependence of the resolution on the shower distance to the detector. We have applied different angular reconstruction methods (AA1, AA2, NE1, NE2, AE1) ${ }^{3}$ for comparison. Obviously, the resolution remains relatively constant with a small increase as function of the distance (Fig. 6). The effect becomes stronger for the outer parts of the FOV. The effective efficiency (comprising trigger- and reconstruction-efficiency) has an almost linear dependence with the radius. Having a high trigger and reconstruction efficiency (about 90\%) in the centre, it drops to almost zero at $500 \mathrm{~km}$ radius. This comes from the decrease of both trigger- and reconstruction efficiency within a radius of about $300 \mathrm{~km}$. In the outer regions it is mainly due to the trigger efficiency. Events

\footnotetext{
${ }^{2}$ see this ICRC's proceedings article: T. Mernik et al., The Angular Resolution of the JEM-EUSO Mission: an Updated View

${ }^{3}$ for details on the algorithms please refer to [7, and references therein]
} 


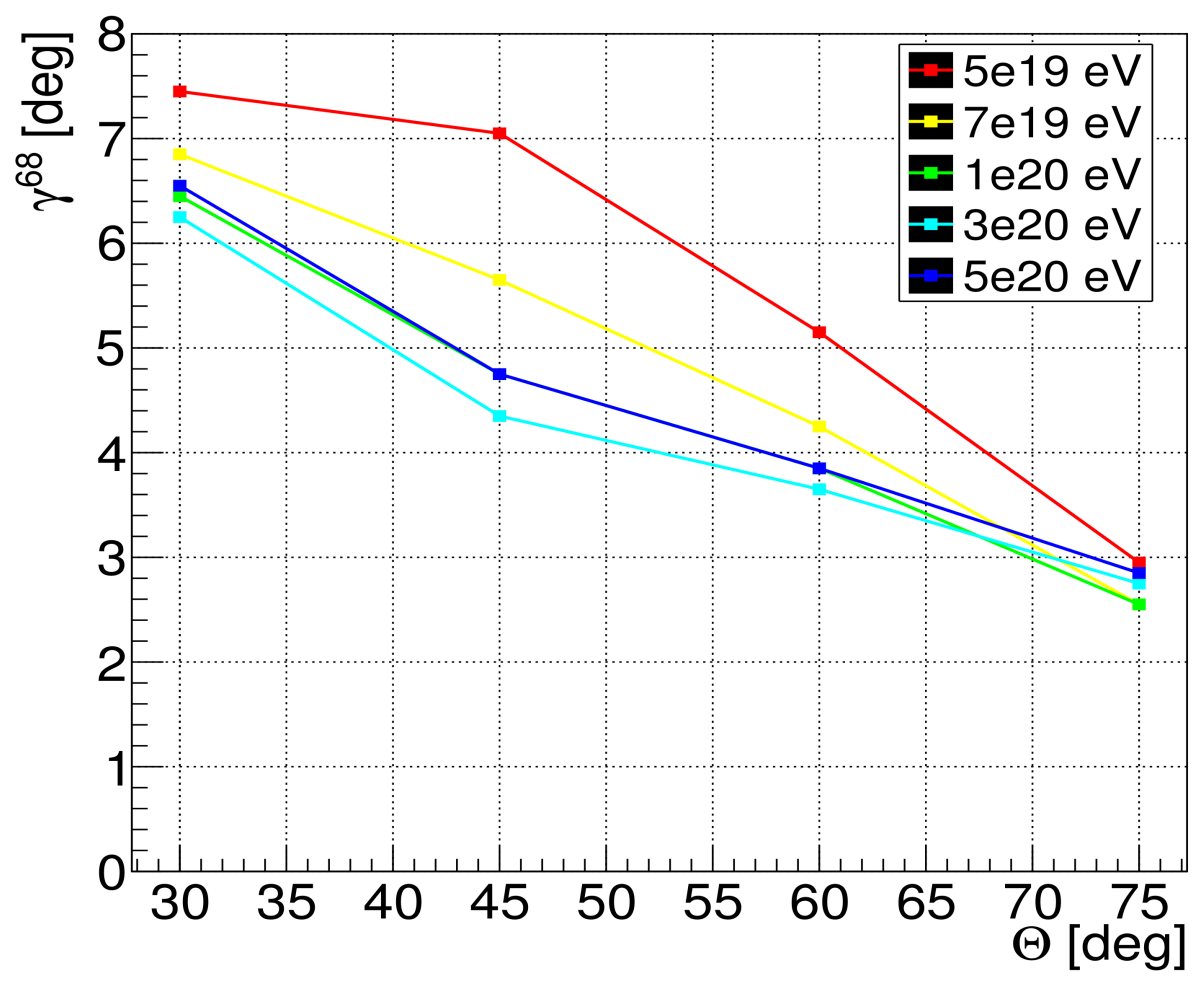

Figure 5: The angular resolution of the tilted (40 $)$ JEM-EUSO telescope [7]. $\gamma$ denotes the angle between the true and the reconstructed direction. $\gamma^{68}$ indicates the cumulative distribution.

sufficiently bright to be triggered can usually also be reconstructed. For the $40^{\circ}$ case we see a relatively constant resolution at $\gamma \approx 4^{\circ}$ within the first $400 \mathrm{~km}$ of the FOV radius. From there to the outer parts of the FOV, it continuously increases up to $\gamma \approx 8^{\circ}$ at $800 \mathrm{~km}$ (Fig. 7). The chaotic behaviour comes from a lack of statistics, since in this region of the FOV, the efficiency has already fallen below $5 \%$. For the $40^{\circ}$ tilting, the detector never reaches a full trigger efficiency, due to a superposition of optics inefficiencies and geometrical reasons.

\section{Conclusion and Outlook}

We studied two tilting cases - a moderate tilting angle of $20^{\circ}$ which enlarges the actual field of view only slightly and a strong tilting angle of $40^{\circ}$. As expected from the analysis of the signal behaviour, we can observe a worsening of the angular resolution due to the fact that less light per CR shower reaches the telescope.

The instrument resolution capability is determined by three limiting factors that are related to the distance of the shower to the detector.

- Proximity effect: Events injected nearby the telescope appear brighter than those farther away, since the amount of photons reaching the telescope is scaled by a factor of $1 / d^{2}$. This is the most dominant contribution. 

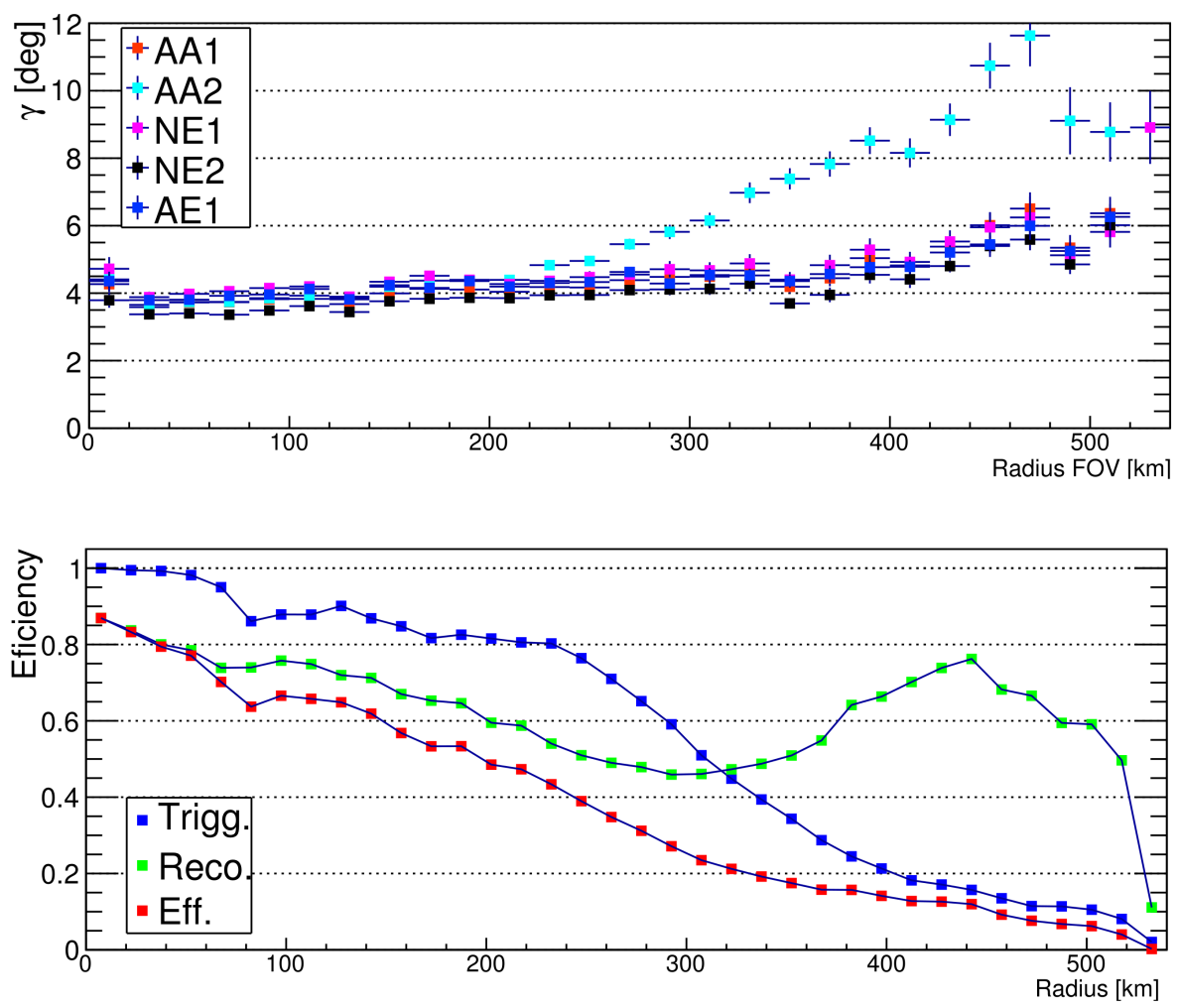

Figure 6: $20^{\circ}$ tilted telescope [7]: The angular resolution expressed in mean $\gamma$ in dependence of the distance from the nadir point (upper). The efficiencies of the trigger, the reconstruction and both together (effective) as function of the radius (lower).

- Projected pixel size on ground: The size of a pixel projected on ground determines the minimum theoretically reachable air shower resolution of the telescope. Pixels in the outer parts of the FOV observe a larger volume of air than the ones in the centre.

- Optics throughput: Events occurring in the outer parts of the FOV face stronger optical losses, due to a lower transmittance of the optical system. The probability of being attenuated or defocussed by the telescope's optics is higher.

The question whether or not to tilt an EUSO-like instrument in space strongly depends on the primary objective of the mission. When the emphasis is put on high exposure a tilting of the instrument might be useful to consider. When the focus lies on accuracy for direction determination the nadir mode is the preferred operation mode.

Acknowledgement: This work was partially supported by Basic Science Interdisciplinary Research Projects of RIKEN and JSPS KAKENHI Grant (22340063, 23340081, and 24244042), by the Italian Ministry of Foreign Affairs, by the 'Helmholtz Alliance for Astroparticle Physics HAP' funded by the Initiative and Networking Fund of the Helmholtz Association, Germany, and by Slovak Academy of Sciences MVTS JEM-EUSO as well as VEGA grant agency project 2/0076/13. Russia is supported by the Russian Foundation for Basic Research Grant No 13-02-12175-ofi-m. The Spanish Consortium involved in the JEM-EUSO Space Mission is funded by MICINN \& MINECO. This work was supported under the ESA Topical Team Contract No. 4000103396 and by the Bundesministerium für Wirtschaft und Technologie through the Deutsches Zentrum für Luft- und Raumfahrt e.V. (DLR) under the grant number FKZ 50 QT 1101. 

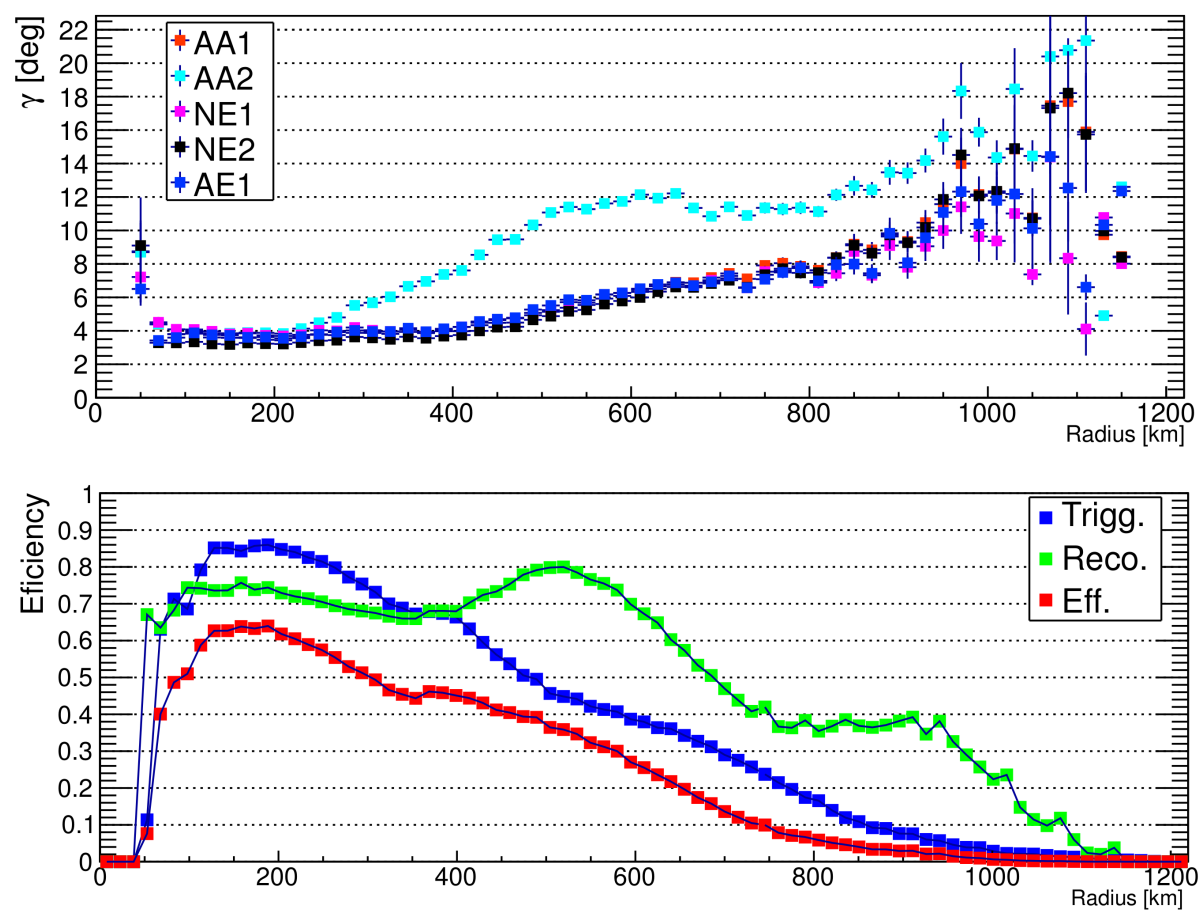

Figure 7: $40^{\circ}$ tilted telescope [7]: The angular resolution expressed in mean $\gamma$ in dependence of the distance from the nadir point (upper). The efficiencies of the trigger, the reconstruction and both together (effective) as function of the radius (lower).

\section{References}

[1] T. Ebisuzaki,et al., The JEM-EUSO Project: Observing Extremely High Energy Cosmic Rays and Neutrinos from the International Space Station, Nucl. Phys. B Proc. Suppl. 175 (2008) 236

[2] L. Wiencke, The JEM-EUSO Mission, APS Meeting Abstracts (2013)

[3] J.H. Adams Jr. et al. - JEM-EUSO Collaboration, An evaluation of the exposure in nadir observation of the JEM-EUSO mission Astroparticle Physics 44 (2013) 76

[4] M. Bertaina and the JEM-EUSO Collaboration, The JEM-EUSO Mission Nuclear Physics B Proceedings Supplements 190 (2009)

[5] Y. Takahashi and The JEM-EUSO Collaboration, The JEM-EUSO mission, New Journal of Physics 11 (2009) 5009

[6] T. Ebisuzaki, Space-based observation of the extensive airshowers, EPJ Web of Conferences $\mathbf{5 3}$ (2013) 1014

[7] T. Mernik, The Expected Angular Resolution of the JEM-EUSO Mission, Dissertation (2014)

[8] A. Thea, C. Berat, S. Bottai et al., The EUSO Simulation and Analysis Framework, Proceedings of the International Cosmic Ray Conference 9 (2005) 133 